\title{
Contributions of unstable and virtual particles to nuclear form factors
}

\author{
M. L. Nekrasov \\ Institute for High Energy Physics, NRC "Kurchatov Institute", \\ Protvino 142281, Russia
}

\begin{abstract}
The coherent meson scattering off heavy nuclei with the production of two particles in the final state is investigated. We obtain the form factors for the direct production of the final state and through intermediate particles, unstable ones that can decay inside the nucleus and virtual ones. The cases of scattering both in the Coulomb and in the strong field of the nucleus are considered. This work is stimulated by an experimental study of the chiral anomaly in a beam of charged kaons in the OKA facility.
\end{abstract}

\section{Introduction}

Reactions of coherent production off nuclei provide important information on the behavior of hadron systems in a nuclear environment and simultaneously on hadron interactions that are difficult or impossible to study in other ways. In particular, they provide a unique opportunity to measure vertices with anomalous parity of the type $K K \pi \gamma$ or $\pi \pi \pi \gamma$, predicted by the effective Wess-Zumino-Witten action [1,2]. These vertices are not available for study in the decay reactions, and their measurement is obstructed by large backgrounds in the proton-target experiments. However, they become available in coherent scattering of charged kaons and pions off nuclei with large atomic numbers owing to the factor $Z^{2}$, where $Z$ is the nuclear charge.

Specifically, we mean reactions of coherent production of $K^{ \pm} \pi^{0}$ or $\pi^{ \pm} \pi^{0}$ off heavy nuclei in the $K^{ \pm}$or $\pi^{ \pm}$beams. At sufficiently high energies and low momentum transfers the Coulomb contributions in these reactions are dominant and can be separated from competing strong contributions [3]. The most favorable transfer is twice its minimum value, when Coulomb contributions reach maximum. In turn, among the Coulomb-type contributions the significance of the anomalous ones increases with decreasing invariant masses of $K^{ \pm} \pi^{0}$ and $\pi^{ \pm} \pi^{0}$. Thus, the favorable domain for determining the anomalous vertices is located at small transfers and small invariant masses of mesons in the final state [4].

In reality, however, the measurements are carried far beyond the mentioned domain, with the excess in the transfer $t$ by 2 orders of magnitude $[6,5]$. As a result, 

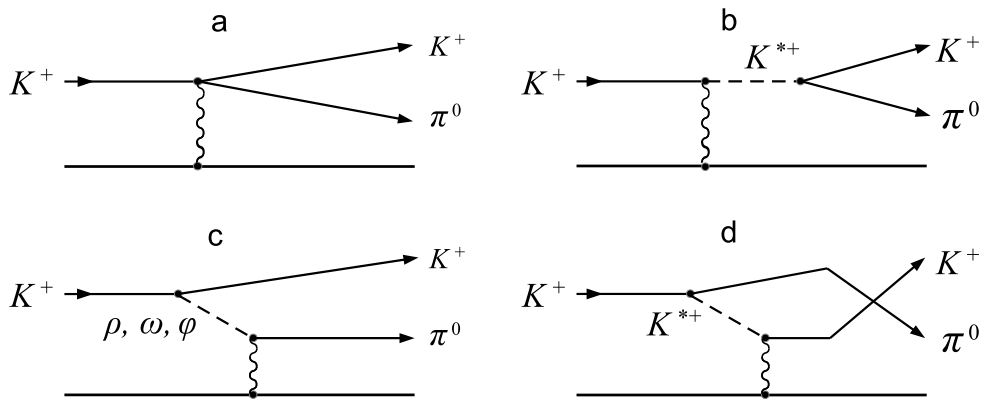

Figure 1: Coherent production of $K^{+} \pi^{0}$ in the $K^{+}$beam in the Coulomb field of nucleus: direct production owing to the chiral anomaly (a), via $K^{*+}$ in the $s$-channel (b), via $\rho, \omega, \phi$ in the $t$-channel (c) and $K^{*+}$ in the $u$-channel (d). The same diagrams describe similar processes due to strong field of the nucleus with the replacement of photon by reggeon.

background contributions become significant. Fig.1 represents relevant diagrams in the case of kaon beam $[7,8,9]$. Preliminary estimates $[9,8]$ show that the background contributions become comparable to the anomaly one shown in Fig.1a. Unfortunately, ambiguities in the form factors prevent more accurate definition of all the contributions. Point is that the data for processing were collected in the transfer region that overlaps the geometric size of the nucleus in configuration space. Specifically, [6] used data at $|t| \leq 0.025(\mathrm{GeV} / \mathrm{c})^{2}$ which means distances $1.2 \mathrm{fm}$ and more with the nucleus radius for $\mathrm{Cu}$ target $4.4 \mathrm{fm}$, and [5] used $|t| \leq 0.025(\mathrm{GeV} / \mathrm{c})^{2}$ which means $1.2 \mathrm{fm}$ with the nucleus radius $2.5-4.2 \mathrm{fm}$ for various targets. In both cases a significant part of inside of the nucleus falls into the measured region. This should significantly affect the scattering in the strong field and could affect the Coulomb scattering (because considerable part of the nuclear surface, where Coulomb forces reach maximum, falls into the interaction region). In the case of real stable particles the way to account for these effects is well understood in Glauber multiple-scattering theory $[10,11,12]$. However, it is not known how to describe the effect in the presence of intermediate particles such as unstable particles that can decay inside the nucleus and especially the virtual particles. Accordingly, a method of determining the form factors in the mentioned cases is not known, too.

In this paper we solve this problem. First of all, we define the form factors in the case of direct production of pairs of particles in the final state. The solution basically follows the extension of Glauber theory to the scattering of composite systems $[13,14]$. The contributions of intermediate unstable particles we consider on the basis of their description as superpositions of quasi-stable particles and their decay products. In the case of virtual particles we use the field-theoretical analysis of coherent scattering of fast particles off nonrelativistic "soft" systems composed of many constituents [15].

We carry out analysis mostly in a general form, but in the case of contributions of the anomaly we turn to the particular reaction $K^{+} A \rightarrow K^{+} \pi^{0} A$, currently investigated in the OKA experiment (IHEP, Protvino) [16]. A theoretical study of this reaction in the context of the mentioned experiment was carried out in $[7,8,9]$. Unfortunately, any details of the interaction of the incident particle with the nucleus were not taken into account, since the form factors were introduced in the unified 
Gaussian form. However, the forms factors are much more complex and their structure depends on the underlying process. In the present paper, we define the forms factors individually for each of the diagrams Fig.1a,b,c,d.

In the next section, we describe the method of our study. The form factor for the direct production of pairs of particles is determined in sect. 3. In sect. 4 and 5 we define the form factors in the presence of unstable and virtual particles. The results are discussed in sect. 6 .

\section{Basic approximation}

The main means for analysis of the collisions of fast particles with nucleus followed by elastic or quasi-elastic scattering at small angles is provided by Glauber multiplescattering theory $[10,11]$. This theory is based on the assumption that the nucleons of the nucleus are "frozen" during the passage of the fast particle, and the impact of each of the nucleons on the incident particle does not depend on the impact of other nucleons. The first condition means that nucleon of the nucleus can be characterized by their positions determined by the wave function. The second condition means additivity of the eikonal phase of the scattered particle.

In this approach, the amplitude of the coherent production of particle $\mathfrak{a}^{*}$ in the $\mathfrak{a}$ beam off a nucleus consisting of $A$ nucleons is determined as follows: ${ }^{1}$

$$
\begin{gathered}
F^{\mathfrak{a} \rightarrow \mathfrak{a}^{*}}(\overrightarrow{\mathbf{q}})=\int \mathrm{d}^{3} \overrightarrow{\mathbf{r}} e^{\mathrm{i} \overrightarrow{\mathbf{q}} \mathbf{r}} \int \prod_{n=1}^{A} \mathrm{~d} \overrightarrow{\mathbf{r}}_{n}\left|\Psi\left(\overrightarrow{\mathbf{r}}_{1}, \ldots, \overrightarrow{\mathbf{r}}_{A}\right)\right|^{2} \\
\times \sum_{n=1}^{A} \prod_{\substack{j=1 \\
j \neq n}}^{A-1}\left[1-\theta\left(z_{j}-z\right) \gamma_{j}^{\mathfrak{a}^{*}}\left(\mathbf{b}-\mathbf{b}_{j}\right)\right] f_{n}^{\mathfrak{a} \rightarrow \mathfrak{a}^{*}}\left(\overrightarrow{\mathbf{r}}-\overrightarrow{\mathbf{r}}_{n}\right) \prod_{\substack{j=1 \\
j \neq n}}^{A-1}\left[1-\theta\left(z-z_{j}\right) \gamma_{j}^{\mathfrak{a}}\left(\mathbf{b}-\mathbf{b}_{j}\right)\right] .
\end{gathered}
$$

Here $\overline{\mathbf{q}}=\left(\mathbf{q}, q_{z}\right)$ is the momentum transferred to the nucleus with $\mathbf{q}$ is its twodimensional component in the impact-parameter plane and axis $z$ is oriented along the direction of motion of the projectile. The longitudinal component is $q_{z}=\left(m_{\mathfrak{a}^{*}}^{2}-\right.$ $\left.m_{\mathfrak{a}}^{2}\right) /(2 k)$ where $m_{\mathfrak{a}^{*}}$ and $m_{\mathfrak{a}}$ are the masses of $\mathfrak{a}^{*}$ and $\mathfrak{a}$, respectively, $k$ is the $\mathfrak{a}$ momentum in the lab frame. The $\Psi$ is the wave function of the nucleus, $\overline{\mathbf{r}}_{j}=\left(\mathbf{b}_{j}, z_{j}\right)$ are the coordinates of the "frozen" nucleons counting from the center of mass of the nucleus (we neglect the effect of the c.m. motion). The $f_{n}^{\mathfrak{a} \rightarrow \mathfrak{a}^{*}}\left(\overline{\mathbf{r}}-\overline{\mathbf{r}}_{n}\right)$ is the amplitude in the coordinate space of the conversion $\mathfrak{a} \rightarrow \mathfrak{a}^{*}$ off $n$-th nucleon, averaged over its isotopic spin and spin (effectively off a spinless nucleon), $\mathbf{r}=(\mathbf{b}, z)$ is a point where the conversion $\mathfrak{a} \rightarrow \mathfrak{a}^{*}$ occurs. The profile functions of elastic scattering before and after the conversion are defined in the standard way,

$$
\gamma_{j}^{\mathfrak{a}\left(\mathfrak{a}^{*}\right)}(\mathbf{b})=\frac{1}{2 \pi \mathrm{i} k} \int \mathrm{d}^{2} \mathbf{q} e^{-\mathrm{i} \mathbf{q} \mathbf{b}} f_{j}^{\mathfrak{a}\left(\mathfrak{a}^{*}\right)}(\mathbf{q}),
$$

\footnotetext{
${ }^{1}$ The given formula defines the leading approximation with a single inelastic conversion. Hereinafter in this section we mainly follow [12].
} 
where $f_{j}^{\mathfrak{a}\left(\mathfrak{a}^{*}\right)}$ is the eikonal amplitude of elastic scattering of $\mathfrak{a}\left(\mathfrak{a}^{*}\right)$ off $j$-th nucleon. All amplitudes are normalized by $\mathrm{d} \sigma / \mathrm{d} \Omega=|F|^{2}$ and defined in the lab frame.

Formula (1) is greatly simplified if we neglect the nucleon correlations and assume that all nucleons are described by the same wave functions. In this case

$$
\left|\psi\left(\overrightarrow{\mathbf{r}}_{1} \ldots \overrightarrow{\mathbf{r}}_{A}\right)\right|^{2}=\prod_{n=1}^{A} \rho\left(\overrightarrow{\mathbf{r}}_{n}\right)
$$

where $\rho\left(\overrightarrow{\mathbf{r}}_{n}\right)$ is the distribution density of a single nucleon,

$$
\int \rho(\overrightarrow{\mathbf{r}}) \mathrm{d}^{3} \overrightarrow{\mathbf{r}}=1
$$

By virtue of (3) and neglecting contributions of order $1 / A$, we can collect the products in (1) to the exponent. Then (1) is reduced to

$$
F^{\mathfrak{a} \rightarrow \mathfrak{a}^{*}}(\overrightarrow{\mathbf{q}})=\int \mathrm{d}^{3} \overrightarrow{\mathbf{r}} e^{\mathrm{i} \overrightarrow{\mathbf{q}} \overrightarrow{\mathbf{r}}}\left[\sum_{n=1}^{A} \int \mathrm{d}^{3} \overrightarrow{\mathbf{r}}^{\prime} f_{n}^{\mathfrak{a} \rightarrow \mathfrak{a}^{*}}\left(\overrightarrow{\mathbf{r}}-\overrightarrow{\mathbf{r}}^{\prime}\right) \rho\left(\overrightarrow{\mathbf{r}}^{\prime}\right)\right] e^{\mathrm{i} \chi_{C}(\mathbf{b})} E^{\mathfrak{a}, \mathfrak{a}^{*}}(\mathbf{b}, z),
$$

where $E^{\mathfrak{a}, \mathfrak{a}^{*}}(\mathbf{b}, z)$ is the attenuation function resulting from the elastic scattering in the strong fields of nucleons before and after the conversion $\mathfrak{a} \rightarrow \mathfrak{a}^{*}$,

$$
\begin{gathered}
E^{\mathfrak{a}, \mathfrak{a}^{*}}(\mathbf{b}, z)= \\
=\exp \left\{-A \int_{-\infty}^{z} \mathrm{~d} z^{\prime} \int \mathrm{d}^{2} \mathbf{b}^{\prime} \gamma^{\mathfrak{a}}\left(\mathbf{b}-\mathbf{b}^{\prime}\right) \rho\left(\mathbf{b}^{\prime}, z^{\prime}\right)-A \int_{z}^{\infty} \mathrm{d} z^{\prime} \int \mathrm{d}^{2} \mathbf{b}^{\prime} \gamma^{\mathfrak{a}^{*}}\left(\mathbf{b}-\mathbf{b}^{\prime}\right) \rho\left(\mathbf{b}^{\prime}, z^{\prime}\right)\right\} .
\end{gathered}
$$

Taking into account the short-range nature of strong interactions, (6) is reduced to

$$
E^{\mathfrak{a}, \mathfrak{a}^{*}}(\mathbf{b}, z)=\exp \left\{-\frac{1}{2} \sigma_{\mathfrak{a}}^{\prime} A T_{-}(\mathbf{b}, z)-\frac{1}{2} \sigma_{\mathfrak{a}^{*}}^{\prime} A T_{+}(\mathbf{b}, z)\right\}
$$

where $T_{ \pm}(\mathbf{b}, z)$ are the thickness functions,

$$
T_{-}(\mathbf{b}, z)=\int_{-\infty}^{z} \mathrm{~d} z^{\prime} \rho\left(\mathbf{b}, z^{\prime}\right), \quad T_{+}(\mathbf{b}, z)=\int_{z}^{\infty} \mathrm{d} z^{\prime} \rho\left(\mathbf{b}, z^{\prime}\right) .
$$

Here we used the optical theorem $f(0)=\mathrm{i} k(4 \pi)^{-1} \sigma^{\prime}$,

$$
\sigma^{\prime}=\sigma_{t o t}(1-\mathrm{i} \alpha), \quad \alpha=\operatorname{Re} f(0) / \operatorname{Im} f(0) .
$$

The $\chi_{C}(\mathbf{b})$ in (5) is the Coulomb phase arising in the case of charged incident particle. It is determined by the same-type expression under the exponent in (6), but with summation over protons and with the Coulomb profile functions. In the case of a positively charged particle and a spherical nucleus, $\chi_{C}(\mathbf{b})$ is $[17]$

$$
\chi_{C}(\mathbf{b})=4 \pi Z \alpha\left[\ln (\mu b) \int_{0}^{b} T\left(b^{\prime}\right) b^{\prime} \mathrm{d} b^{\prime}+\int_{b}^{\infty} \ln \left(\mu b^{\prime}\right) T\left(b^{\prime}\right) b^{\prime} \mathrm{d} b^{\prime}\right],
$$


Here $Z$ is a number of protons, and

$$
T(\mathbf{b})=\int_{-\infty}^{+\infty} \rho(\mathbf{b}, z) \mathrm{d} z .
$$

The $\mu$ in (10) is a dimensional parameter. It does not affect the dependence of $\chi_{C}$ on $\mathbf{b}$, and only defines the additive constant part of the phase which is irrelevant. (We can put $\mu=k$ for definiteness.)

In the case of conversion $\mathfrak{a} \rightarrow \mathfrak{a}^{*}$ due to Coulomb field, summation in (5) goes over the charged protons. So in this case

$$
F_{c}^{\mathfrak{a} \rightarrow \mathfrak{a}^{*}}(\overrightarrow{\mathbf{q}})=Z \int \mathrm{d}^{3} \overrightarrow{\mathbf{r}} e^{\mathrm{i} \overrightarrow{\mathbf{q}} \overrightarrow{\mathbf{r}}}\left[\int \mathrm{d}^{3} \overrightarrow{\mathbf{r}}^{\prime} f_{c}^{\mathfrak{a} \rightarrow \mathfrak{a}^{*}}\left(\overrightarrow{\mathbf{r}}-\overrightarrow{\mathbf{r}}^{\prime}\right) \rho\left(\overrightarrow{\mathbf{r}}^{\prime}\right)\right] e^{\mathrm{i} \chi_{C}(\mathbf{b})} E^{\mathfrak{a}, \mathfrak{a}^{*}}(\mathbf{b}, z),
$$

where $f_{c}^{\mathfrak{a} \rightarrow \mathfrak{a}^{*}}$ is the amplitude of inelastic Coulomb scattering off a proton.

In the case of conversion $\mathfrak{a} \rightarrow \mathfrak{a}^{*}$ due to strong interactions, it is convenient to express the amplitude in terms of profile function for elementary inelastic process. Omitting standard calculations, where the short-range property of strong interactions is used, we arrive at

$$
F_{s}^{\mathfrak{a} \rightarrow \mathfrak{a}^{*}}(\overrightarrow{\mathbf{q}})=A \frac{\mathrm{i} k}{2 \pi} \int \mathrm{d}^{3} \overrightarrow{\mathbf{r}} e^{i \overrightarrow{\mathbf{q}} \overrightarrow{\mathbf{r}}}\left[\int \mathrm{d}^{2} \mathbf{b}^{\prime} \gamma_{s}^{\mathfrak{a} \rightarrow \mathfrak{a}^{*}}\left(\mathbf{b}-\mathbf{b}^{\prime}\right) \rho\left(\mathbf{b}^{\prime}, z\right)\right] e^{\mathrm{i} \chi_{C}(\mathbf{b})} E^{\mathfrak{a}, \mathfrak{a}^{*}}(\mathbf{b}, z) .
$$

Recall that $\overrightarrow{\mathbf{q}}=\left(\mathbf{q}, q_{z}\right), \overrightarrow{\mathbf{r}}=(\mathbf{b}, z)$. The profile function $\gamma_{s}^{\mathfrak{a} \rightarrow \mathfrak{a}^{*}}(\mathbf{b})$ is expressed through the inelastic elementary scattering amplitude in the same way as in (2).

\section{Form factors for direct pair-production}

Based on (12) and (13), one can make a detailed definition of amplitudes for various processes. In the initial work [12] the inelastic process $\mathfrak{a} \rightarrow \mathfrak{a}^{*}$ was considered with the change of spin of incident particle, of the type $K A \rightarrow K^{*} A$, in the approximation of stable $\mathfrak{a}^{*}$. It was also assumed that quantum numbers exchanged are those of the photon. In this case the elementary amplitude is proportional to the transverse component of the transfer and may be written as

$$
f_{c, s}^{\mathfrak{a} \rightarrow \mathfrak{a}^{*}}(\overline{\mathbf{q}})=\mathbf{u q} \phi\left(\overline{\mathbf{q}}^{2}\right) .
$$

Here $\mathbf{u}$ is a vector in the impact-parameter plane, $\phi\left(\overline{\mathbf{q}}^{2}\right)$ is proportional to $1 / \overline{\mathbf{q}}^{2}$ in the case of Coulomb forces and is finite at $\overline{\mathbf{q}}^{2} \rightarrow 0$ in the case of strong interactions. Given this behavior, [12] obtained the amplitudes for the coherent Coulomb and strong production. In this section we determine the analogous formulas in the case of direct production of a pair of particles. For definiteness we consider the case of $K^{+} \pi^{0}$ production in the $K^{+}$beam. However our formulas will be valid for any process $\mathfrak{a} A \rightarrow \mathfrak{a c} A$ with charged $\mathfrak{a}$ if the direct conversion $\mathfrak{a} \rightarrow \mathfrak{a} \mathfrak{c}$ is possible due to the chiral anomaly.

We start with the attenuation function. At first we note that at high energies and small invariant mass of the $K \pi$ system the relative angle between scattering $K$ 
and $\pi$ is very small in the lab frame. As a result, they do not have time to spread over long distances in the impact-parameter plane during the passage of nucleus. In particular, at $18 \mathrm{GeV}$ incident $K$ and the invariant mass of $K \pi$ of the order of the nominal mass of $K^{*}$, the $K$ and $\pi$ have time to spread at the distance of order $1 \%$ of the nucleus radius. This is much smaller than the radius of nuclear forces. So the $K^{+} \pi^{0}$ system inside the nucleus can be considered as a pair of unconnected particles moving in parallel with a common impact parameter. The attenuation function of such a system is formed from the products

$$
\prod_{\substack{j^{\prime}, j^{\prime \prime}=1 \\ j^{\prime} \neq j^{\prime \prime} \neq n}}^{A-1}\left[1-\theta\left(z_{j^{\prime}}-z\right) \gamma_{j^{\prime}}^{K^{+}}\left(\mathbf{b}-\mathbf{b}_{j^{\prime}}\right)\right]\left[1-\theta\left(z_{j^{\prime \prime}}-z\right) \gamma_{j^{\prime \prime}}^{\pi^{0}}\left(\mathbf{b}-\mathbf{b}_{j^{\prime \prime}}\right)\right]
$$

instead of the products of $\left[1-\theta\left(z_{j}-z\right) \gamma_{j}^{\mathfrak{a}^{*}}\left(\mathbf{b}-\mathbf{b}_{j}\right)\right]$ in formula (1). In the approximation (3) and in the case of heavy nucleus, this leads to the attenuation function

$$
E^{K^{+}, K^{+} \pi^{0}}(\mathbf{b}, z)=\exp \left\{-\frac{1}{2} \sigma_{K^{+}}^{\prime} A T_{-}(\mathbf{b})-\frac{1}{2}\left(\sigma_{K^{+}}^{\prime}+\sigma_{\pi^{0}}^{\prime}\right) A T_{+}(\mathbf{b}, z)\right\} .
$$

Note that the cross sections in the second term in braces in (16) are determined with the momenta not equal to $k$, but defined by the kinematics of the corresponding processes.

The averaged elementary amplitude of Coulomb scattering with direct conversion $K^{+} \rightarrow K^{+} \pi^{0}$ due to the chiral anomaly is [8]

$$
f_{c}^{K^{+} \rightarrow K^{+} \pi^{0}}(\overrightarrow{\mathbf{q}}, \ldots)=\alpha \frac{\mathbf{c}(\ldots) \mathbf{q}}{\overrightarrow{\mathbf{q}}^{2}}
$$

Here $\mathbf{c}$ is a vector in the impact-parameter plane, and dots mean kinematic variables that are additional to $\overline{\mathbf{q}}$. Calculating Fourier with respect to $\overline{\mathbf{q}}$ and substituting the result into (12), we get

$$
\begin{gathered}
F_{c}^{K^{+} \rightarrow K^{+} \pi^{0}}(\overrightarrow{\mathbf{q}}, \ldots)= \\
=-\mathrm{i} Z \alpha \int \mathrm{d}^{2} \mathbf{b} \mathrm{d} z e^{\mathrm{i} \mathbf{q} \mathbf{b}+\mathrm{i} q_{z} z}\left[\frac{\mathbf{c}(\ldots) \mathbf{b}}{r^{3}} \int_{0}^{r} \mathrm{~d} y y^{2} \rho(y)\right] e^{\mathrm{i} \chi_{C}(\mathbf{b})} E^{K^{+}, K^{+} \pi^{0}}(\mathbf{b}, z),
\end{gathered}
$$

where $r=\sqrt{\mathbf{b}^{2}+z^{2}}$. Calculating the angular integral, we arrive at

$$
F_{c}^{K^{+} \rightarrow K^{+} \pi^{0}}(\overrightarrow{\mathbf{q}}, \ldots)=f_{c}^{K^{+} \rightarrow K^{+} \pi^{0}}(\overrightarrow{\mathbf{q}}, \ldots) \Phi_{c}^{K^{+}, K^{+} \pi^{0}}(\overrightarrow{\mathbf{q}}),
$$

where $\Phi_{c}^{K^{+}, K^{+} \pi^{0}}$ is the Coulomb form factor, ${ }^{2}$

$$
\begin{gathered}
\Phi_{c}^{K^{+}, K^{+} \pi^{0}}(\overrightarrow{\mathbf{q}})= \\
=2 \pi Z \frac{\overrightarrow{\mathbf{q}}^{2}}{q} \int_{0}^{\infty} b^{2} \mathrm{~d} b J_{1}(b q) \int \mathrm{d} z e^{\mathrm{i} q_{z} z}\left[\frac{1}{r^{3}} \int_{0}^{r} \rho(y) y^{2} \mathrm{~d} y\right] e^{\mathrm{i} \chi_{C}(b)} E^{K^{+}, K^{+} \pi^{0}}(b, z),
\end{gathered}
$$

\footnotetext{
${ }^{2}$ Note that similar formula (3.4) in [12] for the case of single particle production contains inaccuracies: factor $\pi$ is lost and the contribution of imaginary part of $\exp \left(\mathrm{i} q_{z} z\right)$ is ignored.
} 
$J_{1}$ is the Bessel function.

In the case of direct conversion $K^{+} \rightarrow K^{+} \pi^{0}$ due to strong interactions, the elementary amplitude may include two contributions, with normal and abnormal parity of the meson vertices,

$$
f_{s}^{K^{+} \rightarrow K^{+} \pi^{0}}(\overrightarrow{\mathbf{q}}, \ldots)=\phi_{s}^{n}\left(\overrightarrow{\mathbf{q}}^{2}, \ldots\right)+\mathbf{h}(\ldots) \mathbf{q} \phi_{s}^{a}\left(\overrightarrow{\mathbf{q}}^{2}, \ldots\right) .
$$

Here dots mean additional kinematic variables like in (17), and $\mathbf{h}$ is a vector in the impact-parameter plane. In the case of scattering at low energies, $\phi_{s}^{a}$ would imply exchanges by isoscalar vector mesons $(\omega, \phi)$ with anomalous vertex $K K \pi V$. Similarly, $\phi_{s}^{n}$ would imply exchanges by isoscalar axial mesons with normal-parity vertex $K K \pi A$. (Note that pseudoscalar exchanges are forbidden as they imply spin flip of the nucleon, which means the loss of the coherence.) At high energies the contributions in the $t$-channel are reggeized. So $\phi_{s}^{n}$ and $\phi_{s}^{a}$ have a form $[18,19]$

$$
\phi_{s}^{n, a}\left(\overline{\mathbf{q}}^{2}, \ldots\right)=\frac{k}{4 \pi s} \sum_{i} \beta_{i}^{n, a}\left(\overline{\mathbf{q}}^{2}, \ldots\right) \frac{1-e^{-i \pi \alpha_{i}}}{\sin \pi \alpha_{i}}\left(s / s_{0}\right)^{\alpha_{i}},
$$

where $\alpha_{i}$ are the Regge trajectories and $s$ is the corresponding Mandelstam variable. The factor $k /(4 \pi s)$ is due to the normalization of the amplitude adopted in (1).

Substituting (21) into (13), with taking into account (2), and considering that the range of strong interaction is much smaller than the nuclear radius, we get

$$
\begin{gathered}
F_{s}^{K^{+} \rightarrow K^{+} \pi^{0}}(\overrightarrow{\mathbf{q}}, \ldots)=\phi_{s}^{n}(0, \ldots) A \int \mathrm{d}^{2} \mathbf{b} \mathrm{d} z e^{\mathrm{i} \mathbf{q} \mathbf{b}+\mathrm{i} q_{z} z} \rho(b, z) e^{\mathrm{i} \chi_{C}(b)} E^{K^{+}, K^{+} \pi^{0}}(\mathbf{b}, z) \\
+\mathrm{i} \phi_{s}^{a}(0, \ldots) A \int \mathrm{d}^{2} \mathbf{b} \mathrm{d} z e^{\mathrm{i} \mathbf{q} \mathbf{b}+\mathrm{i} q_{z} z}\left[\frac{\mathbf{h}(\ldots) \mathbf{b}}{b} \frac{\partial \rho(b, z)}{\partial b}\right] e^{\mathrm{i} \chi_{C}(b)} E^{K^{+}, K^{+} \pi^{0}}(\mathbf{b}, z) .
\end{gathered}
$$

After the calculation of angular integrals, we obtain

$$
F_{s}^{K^{+} \rightarrow K^{+} \pi^{0}}(\overrightarrow{\mathbf{q}}, \ldots)=\phi_{s}^{n}(0, \ldots) \Phi_{s, n}^{K^{+}, K^{+} \pi^{0}}(\overrightarrow{\mathbf{q}})+\mathbf{h}(\ldots) \mathbf{q} \phi_{s}^{a}(0, \ldots) \Phi_{s, a}^{K^{+} K^{+} \pi^{0}}(\overrightarrow{\mathbf{q}}),
$$

where

$$
\begin{array}{r}
\Phi_{s, n}^{K^{+}, K^{+} \pi^{0}}=2 \pi A \int_{0}^{\infty} b \mathrm{~d} b J_{0}(b q) \int \mathrm{d} z e^{\mathrm{i} q_{z} z} \rho(b, z) e^{\mathrm{i} \chi_{C}(b)} E^{K^{+}, K^{+} \pi^{0}}(b, z), \\
\Phi_{s, a}^{K^{+}, K^{+} \pi^{0}}=-\frac{2 \pi A}{q} \int_{0}^{\infty} b \mathrm{~d} b J_{1}(b q) \int \mathrm{d} z e^{\mathrm{i} q z z} \frac{\partial \rho(b, z)}{\partial b} e^{\mathrm{i} \chi_{C}(b)} E^{K^{+}, K^{+} \pi^{0}}(b, z) .
\end{array}
$$

\section{Unstable particles}

Let us return to the case of conversion $\mathfrak{a} \rightarrow \mathfrak{a}^{*}$, and assume that $\mathfrak{a}^{*}$ is an unstable particle that can decay when passing the nucleus. In this section we discuss how the description of section 2 must be changed in this case. 
First we note that as the decay is spontaneous, its probability can be associated with the path length. So the probability that $\mathfrak{a}^{*}$ being produced at point $z$ reaches $z^{\prime}$, is

$$
w_{\mathfrak{a}^{*}}\left(z^{\prime}-z\right)=\exp \left[-\left(z^{\prime}-z\right) / l\right],
$$

where $l$ is the decay length. In the general case it may be considered as a phenomenological parameter. In vacuum, $l=k /\left(m_{\mathfrak{a}^{*}} \Gamma_{\mathfrak{a}^{*}}\right)$. For simplicity we consider the case when $\mathfrak{a}^{*}$ decays over a single channel $\mathfrak{a}^{*} \rightarrow \mathfrak{b} \mathfrak{c}$. Then the probability of occurrence $\mathfrak{b} \mathfrak{c}$ in the point $z^{\prime}$ is

$$
w_{\mathfrak{b c}}\left(z^{\prime}-z\right)=1-w_{\mathfrak{a}^{*}}\left(z^{\prime}-z\right) .
$$

Next we note that a system that decays after production can be described as a superposition of two states. In our case these are the quasi-stable state $\mathfrak{a}^{*}$ and the orthogonal state of the decay products $\mathfrak{b c}$. Both states are taken with the weights $\sqrt{w_{\mathfrak{a}}}$ and $\sqrt{w_{\mathfrak{b c}}}$, respectively. The amplitude of elastic scattering of such a system is the sum of the amplitudes of elastic scattering of each of its parts with the weights $w_{\mathfrak{a}}$ and $w_{\mathfrak{b} \mathfrak{c}}$. Accordingly, $\gamma^{\mathfrak{a}^{*}}\left(\mathbf{b}-\mathbf{b}^{\prime}\right)$ in (6) in this case is replaced by

$$
w_{\mathfrak{a}^{*}}\left(z^{\prime}-z\right) \gamma^{\mathfrak{a}^{*}}\left(\mathbf{b}-\mathbf{b}^{\prime}\right)+w_{\mathfrak{b} \mathfrak{c}}\left(z^{\prime}-z\right)\left[\gamma_{j}^{\mathfrak{b}}\left(\mathbf{b}-\mathbf{b}_{j}\right)+\gamma_{j}^{\mathfrak{c}}\left(\mathbf{b}-\mathbf{b}_{j}\right)\right] .
$$

In the case of charged particles this construction leads to the same Coulomb factor (10). However, the attenuation is different:

$$
\begin{gathered}
E^{\mathfrak{a}, \mathfrak{a}^{*} \rightarrow \mathfrak{b} \mathfrak{c}}(\mathbf{b}, z)= \\
=\exp \left\{-\frac{1}{2} \sigma_{\mathfrak{a}}^{\prime} A T_{-}(\mathbf{b}, z)-\frac{1}{2} \sigma_{\mathfrak{a}^{*}}^{\prime} A T_{+}^{\mathfrak{a}^{*}}(\mathbf{b}, z)-\frac{1}{2}\left(\sigma_{\mathfrak{b}}^{\prime}+\sigma_{\mathfrak{c}}^{\prime}\right) A T_{+}^{\mathfrak{b} \mathfrak{c}}(\mathbf{b}, z)\right\} .
\end{gathered}
$$

Here $\sigma_{\mathfrak{b}}^{\prime}$ and $\sigma_{\mathfrak{c}}^{\prime}$ are determined at the momenta defined by the kinematics of the process, and $T_{+}^{\mathrm{X}}$ is a modified thickness function $\left(\mathrm{X}=\mathfrak{a}^{*}, \mathfrak{b} \mathfrak{c}\right)$,

$$
T_{+}^{\mathrm{X}}(\mathbf{b}, z)=\int_{z}^{\infty} \mathrm{d} z^{\prime} w_{\mathrm{X}}\left(z^{\prime}-z\right) \rho\left(\mathbf{b}, z^{\prime}\right) .
$$

It is readily seen that at $l \rightarrow \infty$ and $l \rightarrow 0$ formula (30) gives the attenuation functions in the above cases of stable $\mathfrak{a}^{*}$ and the direct conversion $\mathfrak{a} \rightarrow \mathfrak{b} \mathfrak{c}$.

Now we define a place in the formula for the amplitude where the decay vertex $\mathfrak{a}^{*} \rightarrow$ $\mathfrak{b} \mathfrak{c}$ has to make a contribution. The problem is that since the decay is spontaneous the appropriate vertex may appear in any place depending on where the decay occurred. However, on the other hand, the decay vertex contributes necessarily together with the propagator connecting it with the vertex of the last elastic $\mathfrak{a}^{*}$ scattering and with the wave functions of the decay products of $\mathfrak{a}^{*}$. Since the averaged amplitude of elastic scattering is proportional to the unit operator in spin variables and $\mathfrak{a}^{*}$ momentum is constant in the leading approximation, the block of above elements - the propagator of $\mathfrak{a}^{*}$, the decay vertex and the wave functions - may be formally attributed to the initial vertex, where $\mathfrak{a}^{*}$ was formed, i.e. to the vertex of the conversion $\mathfrak{a} \rightarrow \mathfrak{a}^{*}$. Simultaneously the wave function of $\mathfrak{a}^{*}$ at the latter vertex may be attributed to the vertex of the last elastic $\mathfrak{a}^{*}$ scattering, see Fig.2. After performing these formal 


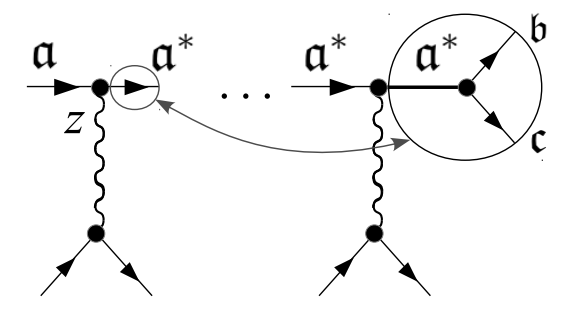

Figure 2: Formal replacement in the elementary amplitudes with the production of particle $\mathfrak{a}^{*}$ and its decay.

manipulations, the result will be the replacement in formula (5) of the amplitude $f_{n}^{\mathfrak{a} \rightarrow \mathfrak{a}^{*}}$ by the amplitude $f_{n}^{\mathfrak{a} \rightarrow \mathfrak{a}^{*} \rightarrow \mathfrak{b} \mathfrak{c}}$ for the cascade process $\mathfrak{a} n \rightarrow \mathfrak{a}^{*} n, \mathfrak{a}^{*} \rightarrow \mathfrak{b} \mathfrak{c}$, where $n$ is the nucleon of the nucleus.

Thus, we arrive at the following formula for amplitude of the entire coherent process:

$$
\begin{gathered}
F^{\mathfrak{a} \rightarrow \mathfrak{a}^{*} \rightarrow \mathfrak{b} \mathfrak{c}}(\overrightarrow{\mathbf{q}}, \ldots)= \\
=\int \mathrm{d}^{3} \overrightarrow{\mathbf{r}} e^{\mathrm{i} \overrightarrow{\mathbf{q}} \overrightarrow{\mathbf{r}}}\left[\sum_{n=1}^{A} \int \mathrm{d}^{3} \overrightarrow{\mathbf{r}}^{\prime} f_{n}^{\mathfrak{a} \rightarrow \mathfrak{a}^{*} \rightarrow \mathfrak{b}, \mathfrak{c}}\left(\overrightarrow{\mathbf{r}}-\overrightarrow{\mathbf{r}}^{\prime}, \ldots\right) \rho\left(\overrightarrow{\mathbf{r}}^{\prime}\right)\right] e^{\mathrm{i} \chi_{C}(\mathbf{b})} E^{\mathfrak{a}, \mathfrak{a}^{*} \rightarrow \mathfrak{b} \mathfrak{c}}(\mathbf{b}, z) .
\end{gathered}
$$

Similar replacements must be made in the formulas that follow (5), including formulas for the form factors.

\section{$5 \quad$ Virtual particles}

If system $\mathfrak{b} \mathfrak{c}$ in the cascade process $\mathfrak{a} \rightarrow \mathfrak{a}^{*} \rightarrow \mathfrak{b} \mathfrak{c}$ is produced far from the $\mathfrak{a}^{*}$ mass shell, then particle $\mathfrak{a}^{*}$ must be virtual, at least immediately before it is converted to the final state. Unfortunately, a priori we do not known at what stage $\mathfrak{a}^{*}$ becomes virtual, and optical analogues do not allow us to understand where this occurs. For this reason, we turn to the field-theoretical analysis of the coherent scattering carried out in monograph [15].

The results of [15] we need are summarized as follows. The scattering of a fast particle off a nonrelativistic "soft" system (nucleus) consisting of $A$ constituents (nucleons) may be represented as a convolution of the product of the wave functions of the "soft" system with the sum of the comb-shaped Green functions, see illustration in Fig.3. The chord of the comb in the Green's functions is made of the propagators of incident particle, and the teeth are the propagators of intermediate particles that couple the incident particle with the constituents of the "soft" system. Among the integration variables one can distinguish the virtualities of the chord propagators. Further, it is assumed that through the chord a large momentum flows, while through the teeth small transfers flow (small-angle scattering that does not destroy the "soft" system). Under these conditions, in integrals over the virtualities one can distinguish a part in which the integration contour may be deformed in such a way that only 


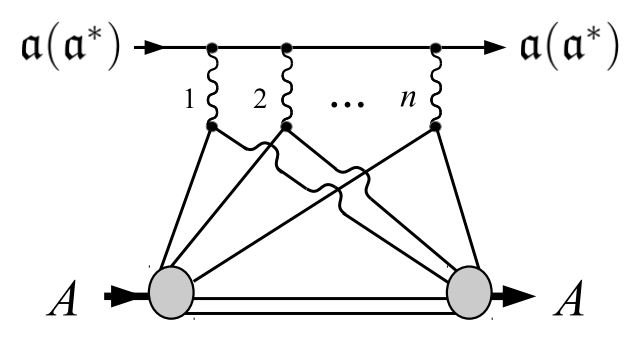

Figure 3: Elastic scattering of a fast particle off a nonrelativistic "soft" system. The scattering off $n$ constituents of the system is shown. The figure is taken from [15].

imaginary part of the propagator of the chord, proportional to $\delta\left(k_{j}^{2}-m^{2}\right)$, makes contributions. Such contributions break down the Green functions into the product of elementary amplitudes. Moreover, their sum forms exactly Glauber approximation.

Simultaneously we know that Glauber approximation is the leading one. From this we deduce that all contributions in the above consideration with the off-shell chord propagators form a correction. At the level of physical processes this means that if a virtual particle appears in the chord, it immediately receives the necessary longitudinal momentum from the constituents and becomes real. Otherwise it determines a correction to the leading approximation.

On this basis we come to the following scenario. After multiple elastic scattering, the incident particle $\mathfrak{a}$ converts in the point $z$ into the on-shell $\mathfrak{a}^{*}$. Then a series of its elastic scattering follows. In the last scattering, say in point $z_{1}, \mathfrak{a}^{*}$ goes off the mass shell, i.e. becomes virtual $\tilde{\mathfrak{a}}^{*}$, and then converts into $\mathfrak{b} \mathfrak{c}$ before interacting with other nucleons. The latter system then elastically scatter.

Since the last $\mathfrak{a}^{*}$ scattering with the conversion $\mathfrak{a}^{*} \rightarrow \tilde{\mathfrak{a}}^{*}$ is accompanied by the mass change, it should be considered as an inelastic process. The generalization of formula (1) to this case is as follows

$$
\begin{aligned}
F^{\mathfrak{a} \rightarrow \widetilde{\mathfrak{a}}^{*} \rightarrow \mathfrak{b} \mathfrak{c}}(\overrightarrow{\mathbf{q}}, \ldots)=- & \int \mathrm{d}^{2} \mathbf{b} \mathrm{d} z e^{\mathrm{i} \mathbf{q} \mathbf{b}+\mathrm{i} q_{z} z}\left[\sum_{n=1}^{A} \int \mathrm{d}^{3} \overrightarrow{\mathbf{r}}^{\prime} f_{n}^{\mathfrak{a} \rightarrow \mathfrak{a}^{*}}\left(\overrightarrow{\mathbf{r}}-\overrightarrow{\mathbf{r}}^{\prime}\right) \rho\left(\overrightarrow{\mathbf{r}}^{\prime}\right)\right] \\
\times \int \mathrm{d} z_{1} \theta\left(z_{1}-z\right) e^{\mathrm{i} q_{z_{1}} z_{1}} & {\left[\sum_{n_{1}=1}^{A-1} \int \mathrm{d}^{3} \overrightarrow{\mathbf{r}}_{1}^{\prime} \frac{2 \pi}{\mathrm{i} k} f_{n_{1}}^{\mathfrak{a}^{*} \rightarrow \widetilde{\mathfrak{a}}^{*} \rightarrow \mathfrak{b} \mathfrak{c}}\left(\mathbf{b}-\mathbf{b}_{1}^{\prime}, z_{1}-z_{1}^{\prime}, \ldots\right) \rho\left(\overrightarrow{\mathbf{r}}_{1}^{\prime}\right)\right] } \\
& \times e^{\mathrm{i} \chi_{C}(\mathbf{b})} E^{\mathfrak{a}, \mathfrak{a}^{*}, \mathfrak{b} \mathfrak{c}}\left(\mathbf{b}, z, z_{1}\right) .
\end{aligned}
$$

Here $\overline{\mathbf{q}}=\left(\mathbf{q}, q_{L}\right)$, and the common minus sign arises due to the shadowing effect caused by the presence of two inelastic processes. With large $k$ the longitudinal transfers $q_{z}$ and $q_{z_{1}}$ are

$$
q_{z}=\frac{m_{\mathfrak{a}^{*}}^{2}-m_{\mathfrak{a}}^{2}}{2 k}, \quad q_{z_{1}}=\frac{m_{\mathfrak{a}^{*}}^{2}-m_{\mathfrak{a}^{*}}^{2}}{2 k^{*}},
$$

where $m_{\widetilde{\mathfrak{a}}^{*}}^{2}=M_{\mathfrak{b} \mathfrak{c}}^{2}$, the invariant mass squared of the $\mathfrak{b} \mathfrak{c}$. Thereby, the total longitudinal transfer is

$$
q_{L}=\frac{m_{\tilde{\mathfrak{a}}^{*}}^{2}-m_{\mathfrak{a}}^{2}}{2 k} .
$$




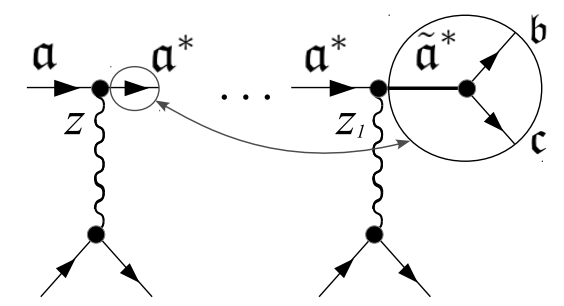

Figure 4: The same as in Fig.2 with virtual $\tilde{\mathfrak{a}}^{*}$.

The attenuation function in (33) is

$$
\begin{gathered}
E^{\mathfrak{a}, \mathfrak{a}^{*}, \mathfrak{b} \mathfrak{c}}\left(\mathbf{b}, z, z_{1}\right)= \\
=\exp \left\{-\frac{1}{2} \sigma_{\mathfrak{a}}^{\prime} A T_{-}(\mathbf{b}, z)-\frac{1}{2} \sigma_{\mathfrak{a}^{*}}^{\prime} A T\left(\mathbf{b}, z, z_{1}\right)-\frac{1}{2}\left(\sigma_{\mathfrak{b}}^{\prime}+\sigma_{\mathfrak{c}}^{\prime}\right) A T_{+}\left(\mathbf{b}, z_{1}\right)\right\},
\end{gathered}
$$

with $T_{-,+}(\mathbf{b}, z)$ defined in $(8)$, and $T\left(\mathbf{b}, z, z_{1}\right)$ is

$$
T\left(\mathbf{b}, z, z_{1}\right)=\int_{z}^{z_{1}} \mathrm{~d} z^{\prime} \rho\left(\mathbf{b}, z^{\prime}\right) .
$$

Recall that $\sigma_{\mathfrak{b}}^{\prime}$ and $\sigma_{\mathfrak{c}}^{\prime}$ are determined at the momenta defined by the kinematics of the process.

The $f_{n_{1}}^{\mathfrak{a}^{*} \rightarrow \widetilde{\mathfrak{a}}^{*} \rightarrow \mathfrak{b} \mathfrak{c}}$ in (33) is the elementary amplitude of the cascade process. It includes, in particular, the propagator of virtual $\widetilde{\mathfrak{a}}^{*}$ and the decay vertex with wave functions. Repeating the reasoning of section 4 , we can formally attribute the above elements to the vertex $\mathfrak{a} \rightarrow \mathfrak{a}^{*}$, and simultaneously attribute the wave function of $\mathfrak{a}^{*}$ at the latter vertex to the vertex $\mathfrak{a}^{*} \rightarrow \widetilde{\mathfrak{a}}^{*}$, see illustration in Fig.4. In doing so, we leave the exponents with the phase shifts in the former places, and we do not change the attenuation function. As a result we arrive at the equivalent formula,

$$
\begin{gathered}
F^{\mathfrak{a} \rightarrow \widetilde{\mathfrak{a}}^{*} \rightarrow \mathfrak{b} \mathfrak{c}}(\overrightarrow{\mathbf{q}}, \ldots)=-\int \mathrm{d}^{2} \mathbf{b} \mathrm{d} z e^{\mathrm{i} \mathbf{q} \mathbf{b}+\mathrm{i} q_{z} z}\left[\sum_{n=1}^{A} \int \mathrm{d}^{3} \overrightarrow{\mathbf{r}}^{\prime} f_{n}^{\mathfrak{a} \rightarrow \widetilde{\mathfrak{a}}^{*} \rightarrow \mathfrak{b} \mathfrak{c}}\left(\overrightarrow{\mathbf{r}}-\overrightarrow{\mathbf{r}}^{\prime}, \ldots\right) \rho\left(\overrightarrow{\mathbf{r}}^{\prime}\right)\right] \\
\times \int \mathrm{d} z_{1} \theta\left(z_{1}-z\right) e^{\mathrm{i} q_{z_{1}} z_{1}}\left[\sum_{n_{1}=1}^{A-1} \int \mathrm{d}^{3} \overrightarrow{\mathbf{r}}_{1}^{\prime} \frac{2 \pi}{\mathrm{i} k} f_{n_{1}}^{\mathfrak{a}^{*} \rightarrow \mathfrak{a}^{*}}\left(\mathbf{b}-\mathbf{b}_{1}^{\prime}, z_{1}-z_{1}^{\prime}\right) \rho\left(\overrightarrow{\mathbf{r}}_{1}^{\prime}\right)\right] \\
\times e^{\mathrm{i} \chi_{C}(\mathbf{b})} E^{\mathfrak{a}, \mathfrak{a}^{*}, \mathfrak{b} \mathfrak{c}}\left(\mathbf{b}, z, z_{1}\right),
\end{gathered}
$$

where $f_{n}^{\mathfrak{a} \rightarrow \widetilde{\mathfrak{a}}^{*} \rightarrow \mathfrak{b c}}$ and $f_{n_{1}}^{\mathfrak{a}^{*} \rightarrow \mathfrak{a}^{*}}$ are the elementary amplitudes of the cascade process and the elastic scattering. Their Fourier by $z$ and $z_{1}$ lead to formally improper longitudinal transfers since we did not rearrange the phase factors. However, this does not affect the full amplitude since the elementary inelastic vertices do not depend on the longitudinal transfers. The dependence on them is contained in the $t$-channel propagators that were not involved in our above manipulations.

Based on (38), we can further refine the definition of the amplitude. Namely, we note that if $\mathfrak{a}^{*}$ is a charged particle, then $f_{n_{1}}^{\mathfrak{a}^{*} \rightarrow \mathfrak{a}^{*}}$ includes two contributions, due 
to strong and Coulomb interactions. In the former case, in view of the short-range nature of strong interactions, the integral $\mathrm{d}^{3} \overline{\mathbf{r}}_{1}^{\prime}$ in (38) is reduced to

$$
V_{s}\left(\mathbf{b}, z_{1}\right)=\frac{1}{2} \sigma_{\mathfrak{a}^{*}}^{\prime} \rho\left(\mathbf{b}, z_{1}\right)
$$

In the case of a long-range Coulomb interaction the integral $\mathrm{d}^{3} \overline{\mathbf{r}}_{1}^{\prime}$ is reduced to the one-fold integral

$$
V_{c}\left(\mathbf{b}, z_{1}\right)=4 \pi \mathrm{i} \alpha\left[\frac{1}{r_{1}} \int_{0}^{r_{1}} \rho(y) y^{2} \mathrm{~d} y+\int_{r_{1}}^{\infty} \rho(y) y \mathrm{~d} y\right] .
$$

Here $r_{1}=\sqrt{\mathbf{b}^{2}+z_{1}^{2}}$, and "i" appears because we take the Coulomb amplitude in the Born approximation, which is real. So, instead of (38) we get

$$
\begin{aligned}
& F^{\mathfrak{a} \rightarrow \widetilde{\mathfrak{a}}^{*} \rightarrow \mathfrak{b} \mathfrak{c}}(\overrightarrow{\mathbf{q}}, \ldots)=-\int \mathrm{d}^{2} \mathbf{b} \mathrm{d} z e^{\mathrm{i} \mathbf{q} \mathbf{b}+\mathrm{i} q_{z} z}\left[\sum_{n=1}^{A} \int \mathrm{d}^{3} \overrightarrow{\mathbf{r}}^{\prime} f_{n}^{\mathfrak{a} \rightarrow \widetilde{\mathfrak{a}}^{*} \rightarrow \mathfrak{b} \mathfrak{c}}\left(\overrightarrow{\mathbf{r}}-\overrightarrow{\mathbf{r}}^{\prime}, \ldots\right) \rho\left(\overrightarrow{\mathbf{r}}^{\prime}\right)\right] \\
& \quad \times \int_{z}^{\infty} \mathrm{d} z_{1} e^{\mathrm{i} q_{z_{1}} z_{1}}\left[(A-1) V_{s}\left(\mathbf{b}, z_{1}\right)+(Z-1) V_{c}\left(\mathbf{b}, z_{1}\right)\right] e^{\mathrm{i} \chi_{C}(\mathbf{b})} E^{\mathfrak{a}, \mathfrak{a}^{*}, \mathfrak{b} \mathfrak{c}}\left(\mathbf{b}, z, z_{1}\right) .
\end{aligned}
$$

Outside the nucleus $V_{c}$ absolutely dominates. Inside, $V_{c}$ and $V_{s}$ may be comparable despite the factor $\alpha$ in (40) because of the factor $4 \pi$ and the big size of the nucleus. Really, the absolute value of $\sigma^{\prime}$ is usually about several tens $\mathrm{mb}$, i.e. several $\mathrm{fm}^{2}$. At distances close to the radius of the nucleus, the square brackets in (40) is roughly estimated as $\rho R^{2} / 3$ with $R \approx A^{1 / 3} \mathrm{fm}$. Hence $\left|V_{c} / V_{s}\right| \approx(8 / 3) \pi \alpha A^{2 / 3}$ which is about 1 if $A=63$, the case of $\mathrm{Cu}$.

Calculating the angular integral in (41), we arrive at the final results. So, in the case of Coulomb scattering, repeating the calculations of sect. 3 we get

$$
F_{c}^{\mathfrak{a} \rightarrow \widetilde{\mathfrak{a}}^{*} \rightarrow \mathfrak{b} \mathfrak{c}}(\overline{\mathbf{q}}, \ldots)=f_{c}^{\mathfrak{a} \rightarrow \widetilde{\mathfrak{a}}^{*} \rightarrow \mathfrak{b} \mathfrak{c}}(\overline{\mathbf{q}}, \ldots) \Phi_{c}^{\mathfrak{a}, \mathfrak{a}^{*}, \mathfrak{b} \mathfrak{c}}(\overline{\mathbf{q}}),
$$

where $\Phi_{c}^{\mathfrak{a}, \mathfrak{a}^{*}, \mathfrak{b} \mathfrak{c}}$ is the Coulomb form factor,

$$
\begin{aligned}
& \Phi_{c}^{\mathfrak{a}, \mathfrak{a}^{*}, \mathfrak{b} \mathfrak{c}}(\overrightarrow{\mathbf{q}})=-2 \pi Z \frac{\overrightarrow{\mathbf{q}}^{2}}{q} \int_{0}^{\infty} b^{2} \mathrm{~d} b J_{1}(b q) \int \mathrm{d} z e^{\mathrm{i} q_{z} z} \frac{1}{r^{3}} \int_{0}^{r} \rho(y) y^{2} \mathrm{~d} y \\
& \times \int_{z}^{\infty} \mathrm{d} z_{1} e^{\mathrm{i} q_{z_{1}} z_{1}}\left[(A-1) V_{s}+(Z-1) V_{c}\right] e^{\mathrm{i} \chi_{C}(b)} E^{\mathfrak{a}, \mathfrak{a}^{*}, \mathfrak{b} \mathfrak{c}}\left(b, z, z_{1}\right) .
\end{aligned}
$$

If the conversion $\mathfrak{a} \rightarrow \mathfrak{a}^{*}$ occurs in the strong field of the nucleus, then the elementary and the full amplitudes are given by (21) and (24), respectively, with the obvious changing in superscripts, and with the form factors

$$
\begin{gathered}
\Phi_{s, n}^{\mathfrak{a}, \mathfrak{a}^{*}, \mathfrak{b} \mathfrak{c}}=-2 \pi A \int_{0}^{\infty} b \mathrm{~d} b J_{0}(b q) \int \mathrm{d} z e^{\mathrm{i} q_{z} z} \rho(b, z) \\
\times \int_{z}^{\infty} \mathrm{d} z_{1} e^{\mathrm{i} q_{z_{1}} z_{1}}\left[(A-1) V_{s}+(Z-1) V_{c}\right] e^{\mathrm{i} \chi_{C}(b)} E^{\mathfrak{a}, \mathfrak{a}^{*}, \mathfrak{b} \mathfrak{c}}\left(b, z, z_{1}\right)
\end{gathered}
$$




$$
\begin{gathered}
\Phi_{s, a}^{\mathfrak{a}, \mathfrak{a}^{*}, \mathfrak{b} \mathfrak{c}}=2 \pi A \frac{1}{q} \int_{0}^{\infty} b \mathrm{~d} b J_{1}(b q) \int \mathrm{d} z e^{\mathrm{i} q_{z} z} \frac{\partial \rho(b, z)}{\partial b} \\
\times \int_{z}^{\infty} \mathrm{d} z_{1} e^{\mathrm{i} q_{z_{1}} z_{1}}\left[(A-1) V_{s}+(Z-1) V_{c}\right] e^{\mathrm{i} \chi_{C}(b)} E^{\mathfrak{a}, \mathfrak{a}^{*}, \mathfrak{b} \mathfrak{c}}\left(b, z, z_{1}\right) .
\end{gathered}
$$

In the particular case of scattering $K^{+} A \rightarrow K^{+} \pi^{0} A$ via virtual $K^{*+}$ in the $s$-channel, one should substitute $K^{+}, K^{*+}, K^{+} \pi^{0}$ for $\mathfrak{a}, \mathfrak{a}^{*}, \mathfrak{b} \mathfrak{c}$ in $(42)-(45)$.

In the end of this section, we consider another scheme of the process with virtual particles, namely $\mathfrak{a} \rightarrow \mathfrak{b} \widetilde{\mathfrak{u}} \rightarrow \mathfrak{b} \mathfrak{c}$, see examples in Fig.1c,d. In this scheme, after the $\mathfrak{a}$ multiple scattering a spontaneous transition $\mathfrak{a} \rightarrow \mathfrak{b} \tilde{\mathfrak{u}}$ occurs with the production of real $\mathfrak{b}$ and virtual $\tilde{\mathfrak{u}}$. Then $\tilde{\mathfrak{u}}$ scatters off a nucleon in the point $z_{1}$, and becomes real $\mathfrak{u}$. The $\mathfrak{b}$ begins elastic scattering at $z_{1}$, as well. After a series of elastic scatterings, $\mathfrak{u}$ converts to $\mathfrak{c}$ in the point $z$, and $\mathfrak{c}$ elastically scatters.

The generalization of the above formulas to this scenario is obvious:

$$
\begin{gathered}
F^{\mathfrak{a} \rightarrow \mathfrak{b} \widetilde{\mathfrak{u}} \rightarrow \mathfrak{b} \mathfrak{c}}(\overrightarrow{\mathbf{q}}, \ldots)=-\int \mathrm{d}^{2} \mathbf{b} \mathrm{d} z_{1} e^{\mathrm{i} \mathbf{q} b+\mathrm{i} q_{z_{1}} z_{1}} \int_{z_{1}}^{\infty} \mathrm{d} z e^{\mathrm{i} q_{z} z}\left[(A-1) V_{s}+(Z-1) V_{c}\right] \\
\times\left[\sum_{n=1}^{A} \int \mathrm{d}^{3} \overrightarrow{\mathbf{r}}^{\prime} f_{n}^{\mathfrak{a} \rightarrow \mathfrak{b} \widetilde{\mathfrak{u}} \rightarrow \mathfrak{b} \mathfrak{c}}\left(\overrightarrow{\mathbf{r}}-\overrightarrow{\mathbf{r}}^{\prime}, \ldots\right) \rho\left(\overrightarrow{\mathbf{r}}^{\prime}\right)\right] e^{\mathrm{i} \chi_{C}(\mathbf{b})} E^{\mathfrak{a}, \mathfrak{b}, \mathfrak{u}, \mathfrak{c}}\left(\mathbf{b}, z_{1}, z\right) .
\end{gathered}
$$

Notice, here $z>z_{1}$ and $V_{s}$ includes $\sigma_{\mathfrak{u}}^{\prime}$ instead of $\sigma_{\mathfrak{a}^{*}}^{\prime}$ in (41). The longitudinal momenta in (46) are as follows

$$
q_{z_{1}}=\frac{m_{\mathfrak{u}}^{2}-m_{\widetilde{\mathfrak{u}}}^{2}}{2 k_{\widetilde{\mathfrak{u}}}}, \quad q_{z}=\frac{m_{\mathfrak{c}}^{2}-m_{\mathfrak{u}}^{2}}{2 k_{\mathfrak{u}}}
$$

where $k_{\widetilde{\mathfrak{u}}}, m_{\widetilde{\mathfrak{u}}}^{2}$ are determined by the kinematics of the process. The attenuation function is

$$
\begin{gathered}
E^{\mathfrak{a}, \mathfrak{b}, \mathfrak{u}, \mathfrak{c}}\left(\mathbf{b}, z_{1}, z\right)=\exp \left\{-\frac{1}{2} \sigma_{\mathfrak{a}}^{\prime} A T_{-}\left(\mathbf{b}, z_{1}\right)\right. \\
\left.-\frac{1}{2} \sigma_{\mathfrak{b}}^{\prime} A T_{+}\left(\mathbf{b}, z_{1}\right)-\frac{1}{2} \sigma_{\mathfrak{u}}^{\prime} A T\left(\mathbf{b}, z_{1}, z\right)-\frac{1}{2} \sigma_{\mathfrak{c}}^{\prime} A T_{+}(\mathbf{b}, z)\right\} .
\end{gathered}
$$

Based on (46) and acting by analogy, the formulas for the processes Fig.1c,d can be easily written. Because of the bulkiness, we do not give them here.

\section{Discussion and conclusion}

In this paper we proceeded from the provision about instantaneous particle formation. Actually this is a common place in Glauber theory. Nevertheless, this is a model assumption, which in a strict sense is not quite correct as the complete formation of particles takes a time. In particular, when converting $\mathfrak{a} \rightarrow \mathfrak{a}^{*}$ this time is of order $q_{z}^{-1}$ in the lab frame. During this time a pre-particle $\mathfrak{a}^{*}$, before it becomes a full-fledged 
particle, passes a distance much larger than the nucleus size. For example, $q_{z}^{-1} \approx 13$ $\mathrm{fm}$ in the case of $K^{*}$ formation in the $18 \mathrm{GeV} K$ beam, while e.g. the $\mathrm{Cu}$ radius is about $4.4 \mathrm{fm}$. In the general case, if $q_{z}^{-1}$ is close to or smaller than the nucleus size, then $q_{z}$ is too large, incompatible with the nucleus integrity. Hence, the $\sigma^{\prime}$ in the attenuation functions actually corresponds to the pre-particle formations rather than real particles. However, in the case of resonances $\sigma^{\prime}$ is practically determined within the Glauber theory framework. This actually eliminates the problem. In other cases, in view of large $q_{z}^{-1}$ the mentioned effect means mainly parametric change of $\sigma^{\prime}$. This can always be taken into account. Thus, the assumption of instantaneous particle formation should not cause severe problems.

Turning to the results of this paper, we recall that our goal was to determine the form factors for the coherent scattering of fast particles off heavy nuclei with the production of pairs of particle in the final state. In the case of the direct pair production we were based on combining the results for coherent inelastic one-particle scattering and elastic scattering of composite systems. If the pair is produced via intermediate particles, the solution depends on whether they are real unstable or virtual ones. In the former case the form factors include the modified thickness functions weighted with the probabilities for survival and decay of the unstable particle. In the case of virtual intermediate particles, we found that they can be virtual only before their first interaction with nucleons of the nucleus or after the last interaction just before they decay to real particles. At other stages fast particles can exist inside the nucleus only as real ones, at least in the leading approximation. The description of the conversion from real to virtual state and vice versa requires an introduction of additional inelastic-scattering vertex, through which the longitudinal component of the momentum is transferred when the virtuality of the particle changes.

Concerning the specific reaction $K^{+} A \rightarrow K^{+} \pi^{0} A$ which is currently being studied at $18 \mathrm{GeV} K^{+}$[16], we obtained the required formulas, but we did not make quantitative estimates. We notice only that in this case our result about unstable particles is of no practical importance, since the decay length of the $K^{*}$ is too long. Namely, it is approximately $85 \mathrm{fm}$ which is to be compared with the radius of the target nucleus of $4.4 \mathrm{fm}$. So the $K^{*}$ should be considered as a stable particle. (At the same time, our results about contributions of virtual particles are fully relevant.) However, at lower energies and wider resonances the situation may change. For instance, in the coherent scattering $\pi A \rightarrow \pi \pi A$ at $2 \mathrm{GeV}$ the decay length of intermediate $\rho$ is about $3.4 \mathrm{fm}$. This means that effect of instability of $\rho$ must be taken into consideration for

most heavy nuclei. The same is the case in other reactions at similar energies with $\rho$ production inside the nucleus.

In general, our study supplements the existing description of the coherent inelastic scattering off heavy nuclei, traced to [12]. The obtained results can be directly applied in the experimental study of the coherent scattering $K A \rightarrow K \pi A$ and $\pi A \rightarrow \pi \pi A$. With appropriate modifications, our results may be applied to other reactions with the pair production off nuclei via intermediate contributions.

\section{Acknowledgement}

The author is grateful to V.F.Obraztsov for proposing the problem of determining 
the form factors for coherent pair-production of particles off heavy nucleus, and to V.S.Burtovoy for useful discussions.

\section{References}

[1] J.Wess, B.Zumino, Phys. Lett. B37 (1971) 95.

[2] E.Witten, Nucl. Phys. B223 (1983) 422.

[3] S.M.Berman, S.D.Drell, Phys.Rev. 133, b791 (1964).

[4] M.V.Terentev, Phys.Lett., B38 (1972) 419.

[5] Yu.M.Antipov et al., Phys.Rev. D36 (1987) 21.

[6] V.S.Burtovoy, Physics of Particles and Nuclei, 48 (2017) 932 [Fiz. Elem. Chast. At. Yadra 48, 890 (2017)].

[7] R.N.Rogalyov, Phys. At. Nucl. 64 (2001) 68 [Yad. Fiz. 64, 72 (2001)].

[8] V.S.Burtovoy, Phys. At. Nucl. 76 (2013) 450 [Yad. Fiz. 76 (2013) 488].

[9] M.I.Vysotsky, E.V.Zhemchugov, Phys. Rev. D93 (2016) 094029.

[10] R.J.Glauber, Lectures in Theoretical Physics, Vol. I, Ed. W.E.Brittin at al. (Wiley Interscience, N.Y., 1959), p. 315.

[11] R.J.Glauber, in Proc. of 2nd Intern. Conf., Rehovoth, 1967, Ed. G.Alexander (North-Holland, Amsterdam, 1967), p. 311.

[12] G.Faeldt, Nucl. Phys. B43 (1972) 591.

[13] W.Czyz, L.C.Maximon, Ann. Phys., 52 (1969) 59.

[14] J.Formanek, Nucl. Phys. B12 (1969) 441.

[15] G.D. Alkhazov, V.V. Anisovich, P.E. Volkovitsky, Diffractive hadron-nucleus interaction at high energies (Nauka, Leningrad, 1991), Chapt. II and III.

[16] E.Ageev et al., www.oka.ihep.ru/Members/zopeadmin/oka-papers/pred.ps/view

[17] R.J.Glauber, in High-energy physics and nuclear structure, Proc. of 3rd Int. Conf., CU, 1969, Ed. S.Devons (Plenum Press, N.Y., 1970) p. 207.

[18] P.D.B. Collins, An Introduction to Regge Theory and High Energy Physics (Cambridge University Press, 1977).

[19] A.C.Irvung, R.P.Worden, Phys. Rep. 34, 119 (1977). 